\title{
Study of Clusters of Defects in Low-cost Digitally Fabricated Frequency Selective Surfaces
}

\author{
Badredin M.Turki ${ }^{1}$, Edward A. Parker ${ }^{1}$, M. Ali Ziai ${ }^{1}$, John C. Batchelor ${ }^{1}$,Veronica Sanchez-Romaguera ${ }^{2}$, Stephen \\ G.Yeates ${ }^{2}$ \\ ${ }^{1}$ School of Engineering and Digital Arts, University of Kent, Canterbury, UK, bmmt2@kent.ac.uk \\ ${ }^{2}$ Organic Materials Innovation Centre (OMIC), School of Chemistry, University of Manchester, UK
}

\begin{abstract}
Additive process fabrication of inexpensive FSS screens might lead to errors in the printing or to damage at installation owing to miss-handling. This paper investigates the introduction of clusters of missing elements in different locations and their effect on the performance of the arrays.
\end{abstract}

Index Terms-Frequency selective surfaces, indoor radio propagation, electromagnetic architecture, inkjet printing.

\section{INTRODUCTION}

Frequency Selective surfaces (FSS) have been in use for several decades in applications such as multiband reflectors in the form of curved secondary mirrors, in multiband feed systems, and as spatial filters used in side lobe suppression and beam forming [1], [2].

The radio spectrum is heavily used, especially the unlicensed bands, which increasingly leads to a degrading in wireless communication especially with channel congestion in indoor environments, and also user privacy may be compromised by eavesdropping by close-by receivers. FSS can provide a reduction of these problems with recent proposals to provide band selective screening within buildings at long wavelength mobile bands to reduce co-channel interference and increase the signal to interference ratio by rejecting interfering signals from adjacent buildings $[3],[4],[5],[6]$.

The process of fabricating large chemically etched copper FSS screens is high cost and requires several process stages if the waste copper is to be reclaimed. Therefore Inkjet technology may be a cost effective means of production for certain scales of production. Drop-on-demand (DoD) print technology deposits precise and repeatable drop sizes achieving the resolutions required for UHF printed circuits on cheap substrates including porous materials such as paper. The performance of an inkjet printed FSS has been reported in [7] where a null depth of about $-35 \mathrm{~dB}$, similar to copper etched FSS, was achieved.

The aim of producing very cheap UHF electromagnetic structures could be achieved by printing on cheap environmentally friendly substrates and also by depositing as few ink layers as possible, given that such FSS can still provide a sufficient level of isolation of about $-20 \mathrm{~dB}$ [8].

In the printing process, errors could occur as a result of imperfections such as ink spray, in which the droplet might break into finer droplets during printing. This could be caused by a partially blocked nozzle. The finer droplets caused by partial blockage could reduce printed edge resolution and close slots in the printed structures. Furthermore, complete nozzle blockage might lead to a total loss of some elements while poor print surface quality, or non-uniformity in the ink sintering process could cause problematic variations in conductivity across the array.

There are also some physical problems during the process of installing arrays on walls, e.g. destroying some of the elements due to miss-handling of the FSS boards or cutting out of some sections for the installation of fixtures and fittings.

Some of these issues such as cuts in elements and the absence of elements were reported previously in [8],[9] and a benchmark of $-20 \mathrm{~dB} \mathrm{~S}_{21}$ null depth was achieved with defects introduced randomly in up to $20 \%$ of the array elements.

This paper investigates the case where elements are totally absent in localized clusters: in arrays of linear dipoles arranged on skewed lattice geometry [10]; linear dipoles arranged on skewed lattice geometry with larger periodicity; and arrays of linear dipoles arranged on square lattice geometry. Clusters of 10 and $20 \%$ of the array size were introduced at the centres and the corners of the panels.

\section{DESIGN}

The complete FSS contained 374 patch dipoles arranged on a skewed lattice [8], with dipole length $L$ and periodicity $P$ equal to 9.4 and $10.4 \mathrm{~mm}$ respectively, horizontal spacing $D_{x}=$ $1 \mathrm{~mm}$, and vertical spacing $D_{y}=2 \mathrm{~mm}$, as shown in Fig. 1 .

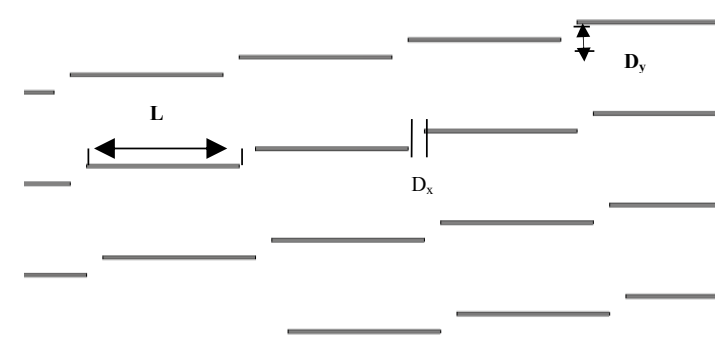

Fig. 1 Skewed lattice dipole FSS

In the larger periodicity design, the elements were also arranged in a skewed lattice with periodicity $P$ now $15.4 \mathrm{~mm}$, horizontal spacing $D_{x}=6 \mathrm{~mm}$, and vertical spacing $D_{y}=5$ $\mathrm{mm}$. Increasing the periodicity led to a decrease in the number of elements within the same physical area to 174 patch dipoles. 
In the square lattice array, the complete FSS contained 475 patch dipole elements with dipole length $L$ and periodicity $P$ equal to 9.4 and $10.4 \mathrm{~mm}$ respectively, as shown in Fig.2.

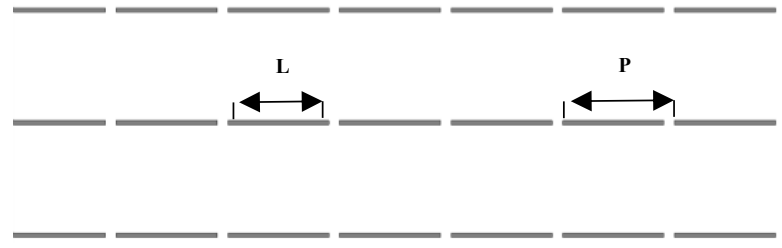

Fig. 2 Square lattice dipole FSS

All FSS arrays were etched onto polyester substrates of $0.045 \mathrm{~mm}$ thickness and relative permittivity $\varepsilon_{\mathrm{r}}=3.5$ with loss tangent $=0.02$. The physical area of the array was $280 \times 190$ $\mathrm{mm}^{2}$.

Each of the four array types was fabricated 4 times, twice missing $10 \%$ and twice missing $20 \%$ of the total elements. The missing dipole cluster sizes were situated either at the centre or at the corner of the FSS. The 4 fabricated cases of the design from Fig.1 are shown in Fig.3.

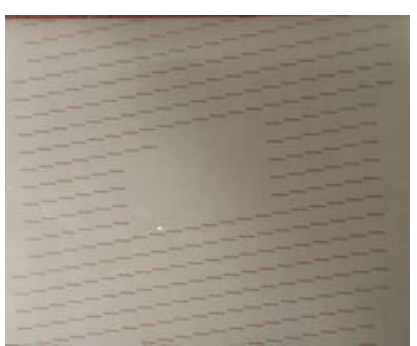

(a)

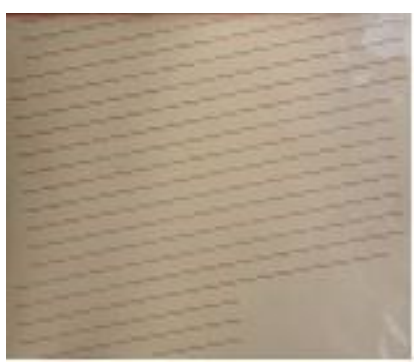

(c)

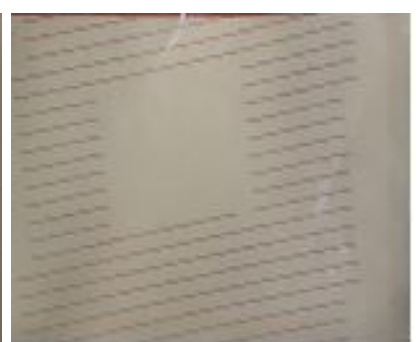

(b)

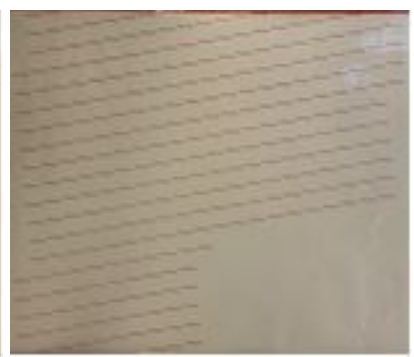

(d)
Fig. 3 Fabricated skewed lattice FSS $(\mathrm{P}=10.4)$ with missing dipole clusters of (a) $10 \%$ at the centre, (b) $20 \%$ at the centre, (c) $10 \%$ at the corner and (d) $20 \%$ at the corner.

\section{RESULTS}

(a) Skewed lattice dipole array, $P=10.4 \mathrm{~mm}$

The random, non-clustered, absence of $10 \%$ and $20 \%$ of the elements across the array as described in [8] led to a reduction in the transmission null depths by 11 and $16 \mathrm{~dB}$ respectively. The measured transmission responses $\left(S_{21}\right)$ for the clustered design differ depending on the position of the missing element clusters. The effect of clustering at the array centre is more pronounced, with a 15 and $20 \mathrm{~dB}$ reduction in the transmission null for the design with $P=10.4$ when 10 and $20 \%$ of the elements were absent, as shown in Fig.4.

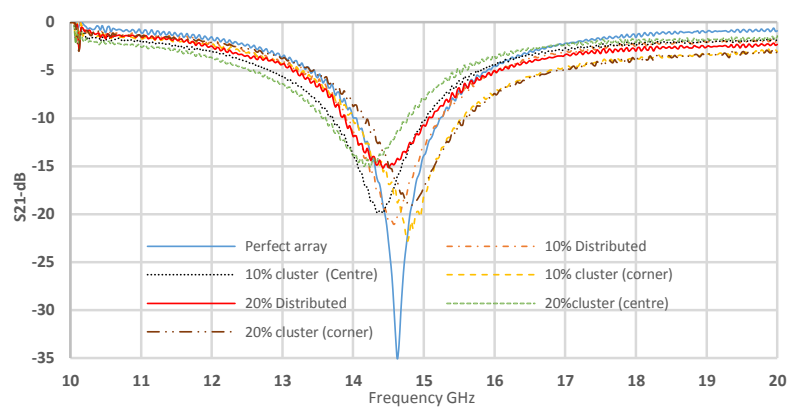

Fig.4 Skewed lattice dipole FSS, $\mathrm{P}=10.4 \mathrm{~mm}$ : measured transmission response $\left(\mathrm{S}_{21}\right)$

(b) Skewed lattice dipole array with increased periodicity, $\mathrm{P}=15.4 \mathrm{~mm}$

The measured $\mathrm{S}_{21}$ of the skewed lattice dipole FSS with larger periodicity $(P=15.4)$ show similar effects to the $P=$ 10.4 design in each of the respective clustering cases. In the $P$ $=15.4$ design, the random non-clustered absence of $10 \%$ and $20 \%$ of the elements reduces the transmission null depths by 9 and $13 \mathrm{~dB}$ respectively compared with the full array. The $S_{21}$ measurements show lower depth of nulls compared with the skewed lattice dipole array with smaller periodicity, about 11 $\mathrm{dB}$ lower in the case of the perfect arrays. The effect of clustering at the array centre, however, is close to the case where the elements were randomly absent and there was 15 and $20 \mathrm{~dB}$ reduction in the transmission null when 10 and 20 $\%$ of the elements were missing, as shown in Fig.5. This is because the larger periodicity reduced the coupling between elements compared with the other designs.

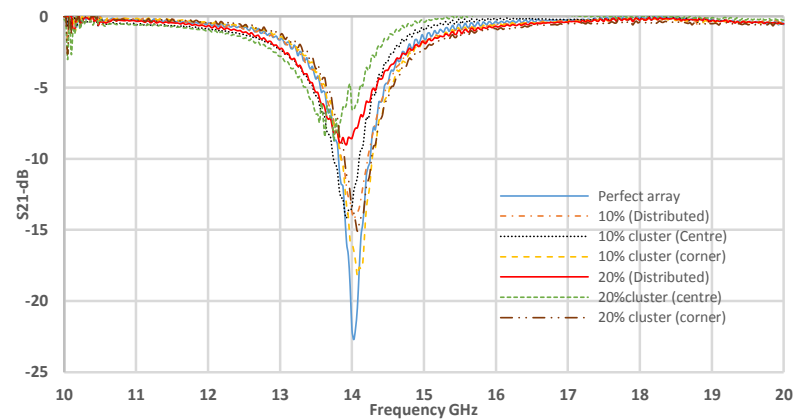

Fig.5 Skewed lattice dipole FSS with larger periodicity, $\mathrm{P}=15.4 \mathrm{~mm}$ : measured transmission response $\left(\mathrm{S}_{21}\right)$

(c) Square lattice dipole array, $P=10.4 \mathrm{~mm}$

The random absence of $10 \%$ and $20 \%$ of the elements across the square lattice dipole array led to decreasing of the transmission null depths by about 8 and $15 \mathrm{~dB}$ respectively [8].

The effect of clustering at the array centre is more pronounced, with a 13 and $21 \mathrm{~dB}$ reduction in the transmission 
null depths when 10 and $20 \%$ of the elements were missing, as shown in Fig.6.

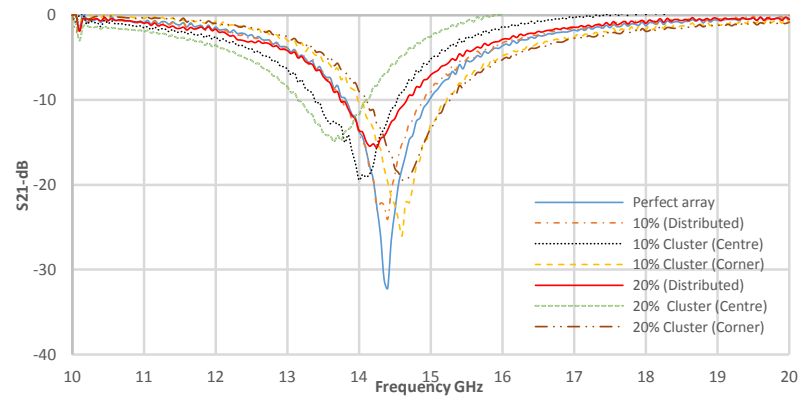

Fig.6 Square lattice dipole FSS, $\mathrm{P}=10.4 \mathrm{~mm}$ : measured transmission response

The clustering effect also causes a shift of about $5-10 \%$ in the resonance frequency, $f_{r}$. The impact of clustering at the corners of the arrays is less apparent than clustering at the centre as illustrated in Table 1.

A similar test was carried out on square loop element arrays and the effect of clustering was similar to the dipole FSS, where the effect was most severe at the centre of the arrays.

\section{CONCLUSIONS}

The effect of introducing randomly located absent and broken elements was discussed in [8], [9], where it was concluded that errors in about $15 \%$ of the elements could be accepted while still achieving a depth of null of -20dB [6]. It now appears that clusters of $10 \%$ missing elements at the centre of the array cause the null depth to be less than $20 \mathrm{~dB}$. However, the deleterious effect of clustering is lower than might be expected, especially for $20 \%$ at the corners of the arrays. This arises from illumination tapering in both the measurements and simulations.

Changes in the illumination profile across an FSS integrated into a wall could be significant in a real building environment due to multipath and varying incidence angles meaning the position of missing element clusters may change in importance with time. Further work is required to understand these issues more fully.

Larger missing element clusters situated in corners were also investigated for the skewed lattice with $P=10.4 \mathrm{~mm}$. In the cases of 30 and $40 \%$ clusters, it was found that the nulls were of only -6 and $-3 \mathrm{~dB}$ respectively meaning they are unlikely to be of practical use.
TABLE 1. Summary of measured results

\begin{tabular}{|c|c|c|c|}
\hline $\begin{array}{c}\text { \% Absent } \\
\text { elements }\end{array}$ & $\begin{array}{c}\text { Skewed } \\
\text { lattice } \\
\text { array, } \\
\mathbf{P}=\mathbf{1 0 . 4 m m}\end{array}$ & $\begin{array}{c}\text { Skewed } \\
\text { lattice } \\
\text { array with } \\
\text { larger }(\boldsymbol{P}), \\
\mathbf{P}=\mathbf{1 5 . 4 m m}\end{array}$ & $\begin{array}{c}\text { Square } \\
\text { lattice } \\
\text { array, } \\
\mathbf{P}=\mathbf{1 0 . 4 m m}\end{array}$ \\
\hline $0 \%$ & -33 & -23 & -31 \\
\hline $10 \%$ (distributed) & -22 & -14 & -23 \\
\hline $10 \%$ at centre & -18 & -13 & -19 \\
\hline $10 \%$ at corner & -22 & -18 & -25 \\
\hline $20 \%$ (distributed) & -17 & -9 & -16 \\
\hline $20 \%$ at centre & -14 & -9 & -13 \\
\hline $20 \%$ at corner & -17 & -14 & -17 \\
\hline
\end{tabular}

\section{ACKNOWLEDGMENT}

We acknowledge the contribution of the UK EPSRC in funding this research through grant EP/J000086/1.

\section{REFERENCES}

[1] J. A. Arnaud, "Resonant-grid quasioptical diplexer," Electron. Lett., vol. 9 , no. 25 , p. $589,1973$.

[2] L. E. Comtesse, R. J. Langley, E. A. Parker, and J. C. Vardaxoglou, "Frequency Selective Surfaces in Dual and Triple Band Offset Reflector Antennas," in 17th European Microwave Conference, 1987, 1987, pp. 208-213.

[3] M. Philippakis, C. Martel, D. Kemp, S. Appleton, R. Pearson, and E.A Parker, "Application of FSS Structures to Selectively Control the Propagation of signals into and out of buildings, Annex 3," Ofcom ref AY4464, pp. 1-54, 2004.

[4] E. A. Parker, J.-B. Robertson, B. Sanz-Izquierdo, and J. C. Batchelor, "Minimal size FSS for long wavelength operation," Electron. Lett., vol. 44, no. 6, p. 394, 2008.

[5] E. A. Parker, J. C. Batchelor, R. Chiang, A. G. Williamson, B Sanz-Izquierdo, M. J. Neve, and K. W. Sowerby, "Frequency selectively screened office incorporating convoluted FSS window," Electron. Lett., vol. 46, no. 5, p. 317, 2010.

[6] A. H. Wong, M. J. Neve, and K. W. Sowerby, "Performance analysis for indoor wireless systems employing directional antennas in the presence of external interference," 2005 IEEE Antennas Propag. Soc. Int. Symp., vol. 1A, pp. 799-802, 2005.

[7] J. C. Batchelor, E. A. Parker, J. A. Miller, V. Sanchez-Romaguera, and S. G. Yeates, "Inkjet printing of frequency selective surfaces," Electron. Lett., vol. 45, no. 1, p. 7, 2009.

[8] B. M. Turki, E. A. Parker, J. C. Batchelor, M. A. Ziai, S. G. Yeates, and V. Sanchez-Romaguera, "Influence of defective elements on performance of frequency selective surfaces," Electron. Lett., vol. 49, no. 17, pp. 1054-1055, Aug. 2013.

[9] B. M. Turki, E. A. Parker, M. A. Ziai, J. C. Batchelor, V. SanchezRomaguera, and S. G. Yeates, "Study of Printing Errors in Digitally Fabricated FSS," Loughborough Conference on Antennas and Propagation (LAPC), November 2013.

[10] S. M. A. Hamdy and E. A. Parker, "Influence of lattice geometry on transmission of electromagnetic waves through arrays of crossed dipoles," IEE Proc. H Microwaves, Opt. Antennas, vol. 129, no. 1, p. 7, 1982 . 